\title{
LINEAR TRANSFORMATIONS ON ALGEBRAS OF MATRICES
}

\author{
MARVIN MARCUS AND B. N. MOYLS
}

1. Introduction. Let $M_{n}$ denote the algebra of $n$-square matrices over the complex numbers; and let $U_{n}, H_{n}$, and $R_{k}$ denote respectively the unimodular group, the set of Hermitian matrices, and the set of matrices of rank $k$, in $M_{n}$. Let $\operatorname{ev}(A)$ be the set of $n$ eigenvalues of $A$ counting multiplicities. We consider the problem of determining the structure of any linear transformation (1.t.) $T$ of $M_{n}$ into $M_{n}$ having one or more of the following properties:
(a) $T\left(R_{k}\right) \subseteq R_{k}$ for $k=1, \ldots, n$.
(b) $T\left(U_{n}\right) \subseteq U_{n}$
(c) $\operatorname{det} T(A)=\operatorname{det} A$ for all $A \in H_{n}$.
(d) $\operatorname{ev}(T(A))=\operatorname{ev}(A)$ for all $A \in H_{n}$.

We remark that we are not in general assuming that $T$ is a multiplicative homomorphism; more precisely, $T$ is a mapping of $M_{n}$ into itself, satisfying

$$
T(a A+b B)=a T(A)+b T(B)
$$

for all $A, B$ in $M_{n}$ and all complex numbers $a, b$.

We shall show first that if $T$ satisfies property (a), then there exist nonsingular matrices $U$ and $V$ such that either

$$
T(A)=U A V
$$

or

$$
T(A)=U A^{\prime} V
$$

for all $A \in M_{n}$, where $A^{\prime}$ is the transpose of $A$. We shall then show that any $T$ satisfying (b), (c), or (d) must in turn satisfy (a), and determine the additional restrictions on $U$ and $V$ required in these cases.

2. Rank Preservers. In this section we shall characterize all linear transformations of $M_{n}$ which preserve rank. To this end it is convenient to consider each matrix of $M_{n}$ as an $n^{2}$-vector, and to represent the 1.t. $T$ as an $n^{2} \times n^{2}$ matrix.

$$
T=\left(\begin{array}{cccc}
T_{11} & T_{12} & \ldots & T_{1 n} \\
\vdots & & \\
\dot{T}_{n 1} & \ldots & T_{n n}
\end{array}\right)
$$

Received March 19, 1958. The work of the first author was partially completed under U.S. National Science Foundation Grant No. NSF-G 5416. The work of the second author was supported in part by the United States Air Force Office of Scientific Research and Development Command. 
where each $T_{i j}$ is an $n$-square matrix. If $v_{j}(A)$ denotes the $j$ th column of $A$, then $T$ maps $A=\left(v_{1}(A), v_{2}(A), \ldots, v_{n}(A)\right)$ into the matrix

$$
\left(\sum_{j=1}^{n} T_{1 j} v_{j}(A), \ldots, \sum_{j=1}^{n} T_{n j} v_{j}(A)\right) \text {. }
$$

Let $\rho(A)$ denote the rank of $A$. If $T$ preserves rank, $T(x, 0, \ldots, 0)=\left(T_{11} x\right.$ $\left.\ldots, T_{n 1} x\right)$ has rank 1 for any non-zero vector $x$ where 0 is the zero vector. We shall call $m n$-square matrices $A_{1}, \ldots, A_{m}$ collinear if, for every nonzero $n$-vector $x$,

$$
\rho\left(A_{1} x, \ldots, A_{m} x\right)=1 .
$$

Lemma 1. If $A_{1}, \ldots, A_{m}$ are collinear, there exist non-zero vectors $z_{1}, \ldots, z_{\text {n }}$ such that

$$
v_{j}\left(A_{i}\right)=k_{i j} z_{j}, \quad i=1, \ldots, m ; j=1, \ldots, n ;
$$

where the $k_{i j}$ are scalars. Moreover, for each $j, k_{i j} \neq 0$ for some $i$.

Proof. Let $e_{j}$ denote the unit vector with $j$ th entry equal to 1 . Then $A_{i} e_{j}=v_{j}\left(A_{i}\right)$. The lemma follows from the fact that $\rho\left(v_{j}\left(A_{1}\right), \ldots, v_{j}\left(A_{m}\right)\right)=1$.

Lemma 2. If the matrices $A_{1}, \ldots, A_{m}$ are collinear, and $z_{1}, z_{\beta}$ are linearly independent for some $\beta$ (cf. (2)), then there exists a non-singular matrix $A$ and scalars $l_{i}$, not all zero, such that

$$
A_{i}=l_{i} A, \quad i=1, \ldots, m
$$

Proof. The matrix $\left(A_{1}\left(e_{1}+e_{\beta}\right), \ldots, A_{m}\left(e_{1}+e_{\beta}\right)\right)=\left(k_{11} z_{1}+k_{1 \beta} z_{\beta}, \ldots\right.$, $\left.k_{m 1} z_{1}+k_{m \beta} z_{\beta}\right)$ has rank 1 . For some $s, k_{s 1} \neq 0$, by Lemma 1 . The Grassmann products

$$
\left(k_{s 1} z_{1}+k_{s \beta} z_{\beta}\right) \wedge\left(k_{i 1} z_{1}+k_{i \beta} z_{\beta}\right)=0,
$$

for $i=1, \ldots, m$. Since $z_{1} \wedge z_{\beta} \neq 0$, it follows that $k_{s 1} k_{i \beta}-k_{s \beta} k_{i 1}=0$, or

$$
k_{i \beta}=\frac{k_{s \beta} k_{i 1}}{k_{s 1}}, \quad i=1, \ldots, m .
$$

Moreover, $k_{s \beta} \neq 0$ (otherwise all $k_{i \beta}=0$ ); and (4) holds for all $\beta$ such that $z_{1}$ and $z_{\beta}$ are independent.

Suppose now that $z_{1}$ and $z_{\gamma}$ are dependent; then $z_{\beta}$ and $z_{\gamma}$ are independent. By the preceding argument,

$$
k_{i \gamma}=\frac{k_{s \gamma} k_{i \beta}}{k_{s \beta}}=\frac{k_{s \gamma}}{k_{s \beta}}\left(\frac{k_{s \beta} k_{i 1}}{k_{s 1}}\right)=\frac{k_{s \gamma} k_{i 1}}{k_{s 1}}, \quad i=1, \ldots, m .
$$

Thus equations (4) hold for all $1 \leqslant \beta \leqslant n$. It follows that $A_{i}=l_{i} A_{s}, i=1$, $\ldots, m$, where $l_{i}=k_{i 1} / k_{s 1}$. In particular $l_{s}=1$.

The matrix $A_{s}$ cannot be singular, for then $\rho\left(A_{1} x, \ldots, A_{m} x\right)=0$ when $x$ is an eigenvector of $A_{s}$ corresponding to the eigenvalue 0 .

An immediate consequence of Lemmas 1 and 2 is 
Lemma 3 . If the matrices $A_{l}, \ldots, A_{n}$ are all singular and collinear, then there exist scalars $k_{i j}$ and a non-zero vector $z$ such that $v_{j}\left(A_{i}\right)=k_{i j} z, i, j=1$, $\ldots, n$.

Lemma t. Let $T$ be a rank preserver on $M_{n}$. If some block $T_{\alpha \beta}$ in the representation (1) of $T$ is non-singular, then there exist scalars $c_{i j}$ such that

$$
T_{i j}=c_{i j} T_{\alpha \beta} ; \quad i, j=1, \ldots, n .
$$

Proof. First note that $T_{1 \beta}, \ldots, T_{n \beta}$ are collinear. Since $T_{\alpha \beta}$ is non-singular, the vectors $z_{1}, \ldots, z_{n}$ of Lemma 1 are linearly independent. Hence $T_{i \beta}=c_{i \beta}$ $T_{\alpha \beta}, i=1, \ldots, n$.

Suppose $T_{\sigma \gamma}$ is also non-singular, $\gamma \neq \beta$. Then $T_{i \gamma}=l_{i \gamma} T_{\sigma \gamma}, i=1, \ldots, n$. If $T_{\sigma \gamma}$ is not a multiple of $T_{\alpha \beta}$, choose a vector $x$ so that $T_{\alpha \beta} x$ and $T_{\sigma \gamma} x$ are linearly independent; and let $X$ be the matrix with $v_{j}(X)=x$ for $j=\beta, \gamma$, and $v_{j}(X)=0$ for $j \neq \beta, \gamma$. Then $\rho(T(X))=1$. This implies that

$$
\left(T_{i \beta} x+T_{i \gamma} x\right) \wedge\left(T_{t \beta} x+T_{t \gamma} x\right)=0, \quad i, t=1, \ldots, n .
$$

Since $T_{\alpha \beta} x \wedge T_{\sigma \gamma} x \neq 0$,

$$
c_{i \beta} l_{t \gamma}-l_{i \gamma} c_{t \beta}=0 \text { for all } i, t .
$$

Let $Y$ be a matrix for which $v_{\beta}(Y)$ and $v_{\gamma}(Y)$ are independent and $v_{j}(Y)=0$ for $j \neq \beta, \gamma$. Then $\rho(Y)=2$, while $\rho(T(Y)) \leqslant 1$ by $(6)$. This contradiction shows that $T_{\sigma \gamma}$ is a multiple of $T_{\alpha \beta}$, and (5) holds for $T_{i \gamma}, i=1, \ldots, n$.

Finally suppose that $T_{i \gamma}$ is singular for some $\gamma$ and all $i$. By Lemma 3 there exist scalars $k_{i j}$ and a non-zero vector $z$ such that $v_{j}\left(T_{i \gamma}\right)=k_{i j} z$. Thus $T_{i \gamma} x$ is a multiple of $z$ for any vector $x$. Choose $y$ so that $T_{\alpha \beta} y=z$, and choose $x$ independent of $y$. For the matrix $Y$ above with $v_{\beta}(Y)=y$ and $v_{\gamma}(Y)=x$, $\rho(Y)=2$, while $\rho(T(Y)) \leqslant 1$. Hence this case cannot arise. This completes the proof of the lemma.

Not every rank preserver need have a non-singular block in its representation (1). For example, the transformation $T_{1}$, which maps each matrix onto its transpose, is linear and preserves rank. In its matrix, $T_{i j}=E_{j i}$, where $E_{i j}$ is the matrix with 1 in the $i, j$ position and 0 's elsewhere. We have, however, the following result.

Lemma 5. Let $T$ be a rank preserver. If every $T_{i j}$ in the representation (1) is singular, then the $n^{2} \times n^{2}$ matrix $T T_{1}$ has a non-singular block.

Proof. By Lemma 3, there exist vectors $z_{1}, \ldots, z_{n}$ such that each column of $T_{i j}$ is a multiple of $z_{j}$ for $i, j=1, \ldots, n$. For any matrix $A, v_{i}(T(A))$ is a linear combination of the columns of the $T_{i j}$. Hence the columns of $T(A)$ are linear combinations of the vectors $z_{j}$. This implies that $z_{1}, \ldots, z_{n}$ are linearly independent; for, if not, the columns of $T(A)$ would be linearly dependent, which is not the case when $A$ is non-singular. Denote the blocks of $T T_{1}$ by $W_{i j}, i, j=1, \ldots, n$. Then 


$$
W_{i j}=\sum_{k=1}^{n} T_{i k} E_{j k}
$$

and $v_{k}\left(W_{i j}\right)=v_{j}\left(T_{i k}\right)$. Thus the $k$ th column of each $W_{i j}$ is a multiple of $z_{k}$. Since $T T_{1}$ preserves rank, the blocks $W_{11}, \ldots, W_{n 1}$ are collinear. The result then follows from Lemma 2.

THEOREM 1. Let $T$ be a l.t. of $M_{n}$ into $M_{n}$. T is a rank preserver if and only if there exist non-singular matrices $U$ and $V$ such that either:

$$
\begin{array}{ll}
T(A)=U A V & \text { for all } A, \\
T(A)=U A^{\prime} V & \text { for all } A .
\end{array}
$$

Proof. The sufficiency of the condition is obvious. For the necessity, if the representation (1) of $T$ has a non-singular block $T_{\alpha \beta}$, choose $U=T_{\alpha \beta}$ and $V=\left(c_{j i}\right)$ in Lemma 4 . If $T$ has no non-singular block, define the rank preserver $T_{2}$ by $T_{2}\left(A^{\prime}\right)=T(A)$. By Lemma $5, T_{2}$ has a non-singular block; hence there exist $U$ and $V$ non-singular such that $T(A)=T_{2}\left(A^{\prime}\right)=U A^{\prime} V$ for all $A$.

3. Determinant Preservers. We shall show that, if a linear transformation $T$ of $M_{n}$ maps unimodular matrices into unimodular matrices, it preserves determinant; that if it preserves determinant, it preserves rank; and determine the appropriate forms of $U$ and $V$ in Theorem 1.

Lemma 6. If the 1.t. $T$ maps $U_{n}$ into $U_{n}$, then $\operatorname{det} T(A)=\operatorname{det} A$ for all matrices $A$.

Proof. If $\operatorname{det} A \neq 0, \operatorname{det}\left[A /(\operatorname{det} A)^{1 / n}\right]=1 ;$ hence $\operatorname{det} T(A)=(\operatorname{det} A)$. $\operatorname{det}\left[T\left(A /(\operatorname{det} A)^{1 / n}\right)\right]=\operatorname{det} A$. Now $\operatorname{det} T(A)$ is a polynomial in the entries $a_{i j}$ of $A$ which is equal to $\operatorname{det} A$ for all non-singular $A$; thus this relation is an identity so that $\operatorname{det} T(A)=\operatorname{det} A$ for all $A$.

Lemma 7. If $T$ preserves determinant, then $T$ is non-singular and hence onto.

Proof. Suppose $T(A)=0$; then $\rho(A)<n$. There exist non-singular matrices $M$ and $N$ such that $M A N=I_{r} \dot{+} 0_{n-r}$, where $r=\rho(A), I_{r}$ is the $r \times r$ unit matrix, $0_{n-r}$ is the $(n-r) \times(n-r)$ zero matrix and $\dot{+}$ denotes the direct sum. For any $X,[\operatorname{det}(M A N+X)] / \operatorname{det} M N=\operatorname{det}\left(A+M^{-1} X N^{-1}\right)=$ $\operatorname{det} T\left(A+M^{-1} X N^{-1}\right)=\operatorname{det} T\left(M^{-1} X N^{-1}\right)=\operatorname{det} M^{-1} X N^{-1}$. Hence $\operatorname{det}(M A N$ $+X)=\operatorname{det} X$. Set $X=0_{r}+I_{n-r}$. Then $\operatorname{det}(M A N+X)=1$, while $\operatorname{det} X$ $=0$ unless $r=0$. Hence $A=0$.

Lemma 8. If $T$ preserves determinant, then $T$ preserves rank.

Proof. Let $A$ be an arbitrary matrix. There exist non-singular matrices $M_{1}, N_{1}, M_{2}, N_{2}$, such that $M_{1} A N_{1}=Y_{1}=I_{r} \dot{+} 0_{n-r}$ and $M_{2} T(A) N_{2}=Y_{2}$ $=I_{s}+0_{n-s}$ where $r=\rho(A)$ and $s=\rho(T(A))$. Define a mapping $\phi$ of $M_{n}$ by:

$$
\phi(X)=M_{2} T\left(M_{1}^{-1} X N_{1}^{-1}\right) N_{2} \text {. }
$$


Then $\phi$ is linear with the property: $\operatorname{det} \phi(X)=k \operatorname{det} X$, where

$$
k=\operatorname{det}\left(M_{2} M_{1}^{-1} N_{1}^{-1} N_{2}\right) ;
$$

also $\phi\left(Y_{1}\right)=Y_{2}$. Set $Y_{3}=0_{r} \dot{+} I_{n-r}$. For any scalar $\lambda$, $\operatorname{det}\left(\lambda Y_{1}+Y_{3}\right)=\lambda^{r}$. On the other hand, $\operatorname{det} \phi\left(\lambda Y_{1}+Y_{3}\right)=\operatorname{det}\left(\lambda Y_{2}+\phi\left(Y_{3}\right)\right)=p(\lambda)$, a polynomial in $\lambda$ of degrees $\leqslant s$. Since $p(\lambda) \equiv k \lambda^{r}, k \neq 0$, identically in $\lambda$, it follows that $r \leqslant s$, and $\rho(A) \leqslant \rho(T(A))$.

By Lemma $7, T^{-1}$ exists; moreover, since $T$ preserves determinant, $\operatorname{det} B$ $=\operatorname{det}\left(\mathrm{TT}^{-1}(B)\right)=\operatorname{det} T^{-1}(B)$ for all $B$ in $M_{n}$. Thus $T^{-1}$ preserves determinant, and $\rho(T(A)) \leqslant \rho\left(T^{-1} T(A)\right)=\rho(A)$. Therefore $\rho(A)=\rho(T(A))$.

Theorem 2. Let $T$ be a 1.t. of $M_{n}$. The following conditions are equivalent:

(i) $T$ maps $U_{n}$ into $U_{n}$.

(ii) T preserves determinant.

(iii) There exist unimodular matrices $U$ and $V$ such that either (7) or (8) holds.

Proof. Lemma 6 gives (i) $\leftrightarrow$ (ii); (iii) $\rightarrow$ (ii) is obvious. If $T$ preserves determinant, then by Lemma 8 and Theorem 1 , there exist non-singular matrices $U_{1}$ and $V_{1}$ such that $T(A)=U_{1} A V_{1}$ or $T(A)=U_{1} A^{\prime} V_{1}$. Since $\operatorname{det} T(I)=1$, $\operatorname{det} U_{1} V_{1}=1$. Choose $U=U_{1} /\left(\operatorname{det} U_{1}\right)^{1 / n}$ and $V=V_{1} /\left(\operatorname{det} V_{1}\right)^{1 / n}$. Thus (ii) $\rightarrow($ iii).

We shall show in the next section that preservation of determinant for Hermitian matrices is also equivalent to conditions (i)-(iii).

\section{Eigenvalue Preservers.}

Lemma 9. Let $T$ be a 1.t. of $M_{n}$. If $\mathrm{ev}(T(H))=\mathrm{ev}(H)$ for all Hermitian matrices $H$, then $\operatorname{ev}(T(A))=\operatorname{ev}(A)$ for all $A$ in $M_{n}$.

Proof. Note first that if $H$ is Hermitian and satisfies the given condition, then $\operatorname{tr}\left\{[T(H)]^{m}\right\}=\operatorname{tr}\left\{H^{m}\right\}$ for $m=1,2, \ldots$, where $\operatorname{tr}(X)$ denotes the trace of $X$. For any matrix $A$ there exist Hermitian matrices $K, L$ such that $A=K+i L$. For real $\alpha, K+\alpha L$ is Hermitian and

$$
\operatorname{tr}\left\{[T(K+\alpha L)]^{m}\right\}=\operatorname{tr}\left\{(K+\alpha L)^{m}\right\} .
$$

For each $m$, equation (9) is a polynomial equation in $\alpha$ of degree $\leqslant m$ satisfied by all real $\alpha$. Hence (9) is satisfied by all complex $\alpha$, and in particular by $\alpha=i$. If the eigenvalues of $A$ and $T(A)$ are $\lambda_{j}$ and $\mu_{j}$, respectively, $j=1, \ldots, n$, then

$$
\sum_{j=1}^{n} \lambda_{j}^{m}=\sum_{j=1}^{n} \mu_{j}^{m} \quad m=1,2, \ldots
$$

It follows that the corresponding elementary symmetric functions of the $\lambda_{j}$ and the $\mu_{j}$ are equal, and that $\operatorname{ev}(T(A))=\operatorname{ev}(A)$. 
Lemma 10. If $\operatorname{ev}(T(A))=\operatorname{ev}(A)$ for all $A \in M_{n}$, then $T(I)=I$, where $I$ is the unit matrix of order $n$.

Proof. $T$ preserves determinant. Hence, for $\lambda$ complex and $A \in M_{n}$, $\operatorname{det}(\lambda I-A)=\operatorname{det}(\lambda T(I)-T(A))=\operatorname{det}(\lambda I-C T(A))$, where $C=(T(I))^{-1}$. Thus ev $(T(A))=\operatorname{ev}(A)=\operatorname{ev}(C T(A))$. Since $T$ is non-singular by Lemma 7 , $T(A)$ ranges over all of $M_{n}$ as $A$ does. Hence $\operatorname{ev}(A)=\operatorname{ev}(C A)$ for all $A$. Choose $U$ unitary so that $C U=H$, where $H$ is positive definite Hermitian. Then $\operatorname{ev}(U)=\operatorname{ev}(C U)=\operatorname{ev}(H)$, so that $U$ has positive eigenvalues. Hence $U=1$ and $C=H$. Since the eigenvalues of $C=(T(I))^{-1}$ are all $1, C=I$, $C^{-1}=I$, and $T(I)=I$.

From Lemmas 9 and 10 and Theorem 2 we obtain

THeOrem 3. Let $T$ be a 1.t. of $M_{n}$. The following conditions are equivalent:

(i) $T$ preserves eigenvalues for all Hermitian matrices in $M_{n}$.

(ii) $T$ preserves eigenvalues for all matrices in $M_{n}$.

(iii) There exists a unimodular matrix $U$ such that either $T(A)=U A U^{-1}$ for all $A \in M$ or $T(A)=U A^{\prime} U^{-1}$ for all $A \in M_{n}$.

TheOREM 4. Let $T$ be a 1.t. of $M_{n}$. If $\operatorname{ev}(T(H))=\operatorname{ev}(H)$ and $T(H)$ is Hermitian for all Hermitian $H$ in $M_{n}$, then the matrix $U$ in Theorem 3 (iii) is unitary.

Proof. $T(H)=(T(H))^{*}$ implies $U H U^{-1}=U^{-1 *} H U^{*}$ and $U^{*} U H=H U^{*} U$. for all Hermitian $H$. It follows easily that $U^{*} U=I$.

TheOREM 5. Let $T$ be a l.t. of $M_{n}$. Then $T$ preserves determinant if and only if it preserves determinant for Hermitian matrices.

Proof. Define $\phi(A)=C T(A)$, where $C=(T(I))^{-1}$. If $T$ preserves determinant for Hermitian $H$, then $\operatorname{det}(\lambda I-H)=\operatorname{det}(\lambda T(I)-T(H))=\operatorname{det}$ $(\lambda I-\phi(H))$ for all real $\lambda$. Hence $\operatorname{ev}(\phi(H))=\operatorname{ev}(H)$, and by Lemma 9, $\operatorname{ev}(\phi(A))=\operatorname{ev}(A)$ for all $A$. Thus $\operatorname{det} A=\operatorname{det} \phi(A)=\operatorname{det} \mathrm{T}(A)$ for all $A$.

Professor N. Jacobson communicated to us the following information while this paper was in press: Theorem 1 was obtained by L. K. Hua (Science Reports of the National Tsing Hua University, Ser. A, 5 (1948) pp. 150-81) and in more general form by H. Jacob (Amer. J. Math., 77 (1955) pp. 177-89). In both these papers $T$ is assumed non-singular; actually our proof of Theorem 1 requires only that $T\left(R_{i}\right) \subseteq R_{i}$, for $i=1,2, n$ without the assumption that $T$ be non-singular. Also Dieudonné (Archiv. d. Math., 1 (1948) pp. 282-7) shows that if $T$ preserves the cone $\operatorname{det} A=0$ and $T$ is non-singular then $T$ has the form indicated in Theorem 2 (iii). Again, our result does not require the assumption that $T$ be non-singular: this follows if $T$ preserves all determinants (Lemma 7).

University of British Columbia 